\title{
Digital Tools Fill a Gap in Mental Health Screening and Support, Particularly for Women Lacking Strong Social Networks
}

\author{
Dani Bradley ${ }^{1}$, MPH, MS; Christina Cobb ${ }^{1}$, MPH, MS; Adam Wolfberg ${ }^{1,2}$, MD, MPH
}

\author{
${ }^{1}$ Ovia Health, Bosotn, MA, United States \\ ${ }^{2}$ Newton Wellesley Hospital, Newton, MA, United States
}

\section{Corresponding Author:}

Dani Bradley, MPH, MS

Ovia Health

308 Congress Street

Boston, MA

United States

Phone: 7817711711

Email: dani.bradley@oviahealth.com

\begin{abstract}
Background: Roughly $11 \%$ of women suffer from postpartum depression nationwide; however, many believe the condition to be widely underreported, in part due to inadequate screening and stigma associated with the condition. Social support networks can help to prevent or mitigate symptoms related to postpartum depression. Single mothers tend to suffer from this condition at a higher rate than married women as they tend to have weaker social networks compared to married women.

Objective: The primary objective ws to determine whether gaps exist in mental health screening and whether digital screening tools can help to fill these gaps. The secondary objective ws to determine whether digitally delivered support proves to be more or less beneficial to subsets of women, namely based on their marital status.

Methods: A survey about mental health history, support, experience with mental health screeners, and characteristics of social networks was sent by email to users of the Ovia Fertility, Ovia Pregnancy, and Ovia Parenting mobile apps. Respondents were all 18 years of age or older and living in the United States. The study was granted exemption by our institutional review board.

Results: Of the 2016 respondents, 39\% reported that they were never screened by their healthcare provider for mental health conditions ( $26 \%$ of women with children and $52 \%$ of women without children). Among women who reported never being screened by a healthcare provider, 17\% reported that they have completed at least one of the screeners (PHQ-9 or Edinburgh Postnatal Depression Scale [EPDS]) in an Ovia mobile app. Of the 2016 respondents, 86\% reported being married or in a domestic partnership. Among the single respondents, $32 \%$ reported either having children, being pregnant, or currently trying to conceive. More single women who have children, are pregnant, or are actively trying to conceive reported that they would feel most supported by a mobile appl (namely, one of Ovia Health's three mobile apps) and to seek treatment for mental health concerns compared to married women (19\% compared to $14 \%$ of married women; $P=.03$ ). Additionally, single women who have children, are pregnant, or are actively trying to conceive reported more often than married women that they feel their mental health is best supported by a mobile appl (16\% compared to $10 \%$ of married women; $P=.007)$. However, both groups of women selected their healthcare provider and their friends/family as the first and second ranking support systems for both seeking mental health treatment and for mental health related support, with the mobile app ranking last.

Conclusions: Screening for mental health conditions during the reproductive health journey is lacking. Digital solutions that deliver clinically validated screening tools help to screen women who are missed in a clinical setting. Women who report being single throughout parenting, pregnancy, or while trying to conceive find more value in mobile app-provided mental health support compared to married women. These findings highlight two gaps that digital technologies, like Ovia Health, can fill: low mental health screening rates during reproductive years and suboptimal social systems.
\end{abstract}

(iproc 2019;5(1):e15207) doi: $\underline{10.2196 / 15207}$

\section{KEYWORDS}

digital screening; mental health; reproductive health; digital support 
Edited by R Palacholla; this is a non-peer-reviewed article. Submitted 26.06.19; accepted 14.08.19; published 02.10.19.

Please cite as:

Bradley D, Cobb C, Wolfberg A

Digital Tools Fill a Gap in Mental Health Screening and Support, Particularly for Women Lacking Strong Social Networks iproc 2019;5(1):e15207

URL: http://www.iproc.org/2019/1/e15207/

doi: $\underline{10.2196 / 15207}$

PMID:

(CDani Bradley, Christina Cobb, Adam Wolfberg. Originally published in Iproceedings (http://www.iproc.org), 02.10.2019 This is an open-access article distributed under the terms of the Creative Commons Attribution License (https://creativecommons.org/licenses/by/4.0/), which permits unrestricted use, distribution, and reproduction in any medium, provided the original work, first published in Iproceedings, is properly cited. The complete bibliographic information, a link to the original publication on http://www.iproc.org/, as well as this copyright and license information must be included. 